\title{
Effects of Orthogonal Rotating Electric Fields on Electrospinning Process
}

\author{
M. Lauricella ${ }^{1}$ a) F. Cipolletta ${ }^{1}$, G. Pontrelli ${ }^{1}$, D. Pisignano ${ }^{2,3}$, and S. Succi ${ }^{1,4 b)}$ \\ ${ }^{1}$ Istituto per le Applicazioni del Calcolo CNR, Via dei Taurini 19, IT 00185 Rome, \\ Italy \\ ${ }^{2}$ Dipartimento di Matematica e Fisica "Ennio De Giorgi", University of Salento, \\ via Arnesano, IT 73100 Lecce, Italy \\ ${ }^{3}$ NEST, Istituto Nanoscienze-CNR, Piazza S. Silvestro 12, IT 56127 Pisa, \\ Italy and \\ ${ }^{4}$ Institute for Applied Computational Science, Harvard "John A. Paulson" \\ School of Engineering and Applied Sciences, Cambridge, MA 02138, \\ United States
}

\begin{abstract}
Electrospinning is a nanotechnology process whereby an external electric field is used to accelerate and stretch a charged polymer jet, so as to produce fibers with nanoscale diameters. In quest of a further reduction in the cross section of electrified jets hence of a better control on the morphology of the resulting electrospun fibers, we explore the effects of an external rotating electric field orthogonal to the jet direction. Through intensive particle simulations, it is shown that by a proper tuning of the electric field amplitude and frequency, a reduction of up to a $30 \%$ in the aforementioned radius can be obtained, thereby opening new perspectives in the design of future ultra-thin electrospun fibers. Applications can be envisaged in the fields of nanophotonic components as well as for designing new and improved filtration materials.
\end{abstract}

Keywords: electrified jets, electric field, electrospinning, computational modelling, nanofibers.

\footnotetext{
a) First author: m.lauricella@iac.cnr.it

b) Corresponding author: s.succi@iac.cnr.it
} 


\section{INTRODUCTION}

Electrospinning has witnessed a dramatic upsurge of interest in recent years because of its potential to produce ultra-fine fibers with sub-micrometer diameters (see Refs 17). Though routinely realizable in the laboratory, electrospinning is a complex phenomenon to analyze because of the coupling between the electric field and the non-linear deformation of the fluid, the latter being dictated by the rheology of the material. As a consequence, the resulting jet (fiber) diameter is affected by several material, design, and operating parameters.

In this context, computational models represent a useful tool to investigate the underlying physics of electrospinning and provide information which may be used for the design of new electrospinning experiments and nanofibrous materials. Several strategies have been pursued to model the process, which can be broadly classified within two main families, Eulerian and Lagrangian. The former is based on a fixed-grid discretization of the partial differential equations of continuum fluid-dynamics ${ }^{8}$ 11, while in the latter the grid moves with the flow, taking the form of particle-like ordinary differential equations. 12

By using suitable theoretical models, the effects of the parameters on the fiber diameter can be systematically studied and assessed, both analytically and numerically. For example, it has been shown that bending (or whipping) instabilities of electrical and hydrodynamical nature, are mostly responsible for jet stretching during the electrospinning process. $12[16 \mid 17$ This behavior leads to a reduction of the cross section radius of electrospun nanofibers. In other studies, the attention is mostly focused on the morphological aspects, revealing a wide variety of pattern depositions of electrified jets. $\frac{16 \mid 18}{1 n}$ the literature, one can find theoretical models to describe the jet dynamics and control of the fiber diameter, through numerical simulations based on multi-parameter choice, involving the perturbation at the nozzle, the intensity of the fixed electric field, the density of the polymer solution. ${ }^{12|18| 19}$ However, investigating new strategies to improve the overall control on electrospun nanofibrous materials, still is an open scientific and technological challenge. In the present work, we consider the effect of a rotating electric field orthogonal to the main electric field (Figure 1). In particular, we present a theoretical model and ensuing numerical simulations with the JETSPIN $\operatorname{code} \mathrm{C}^{20121}$, in order to identify the optimal values of the amplitude and rotational frequency of the orthogonal rotating electric field (OREF), which permit to alter fiber morphology. 


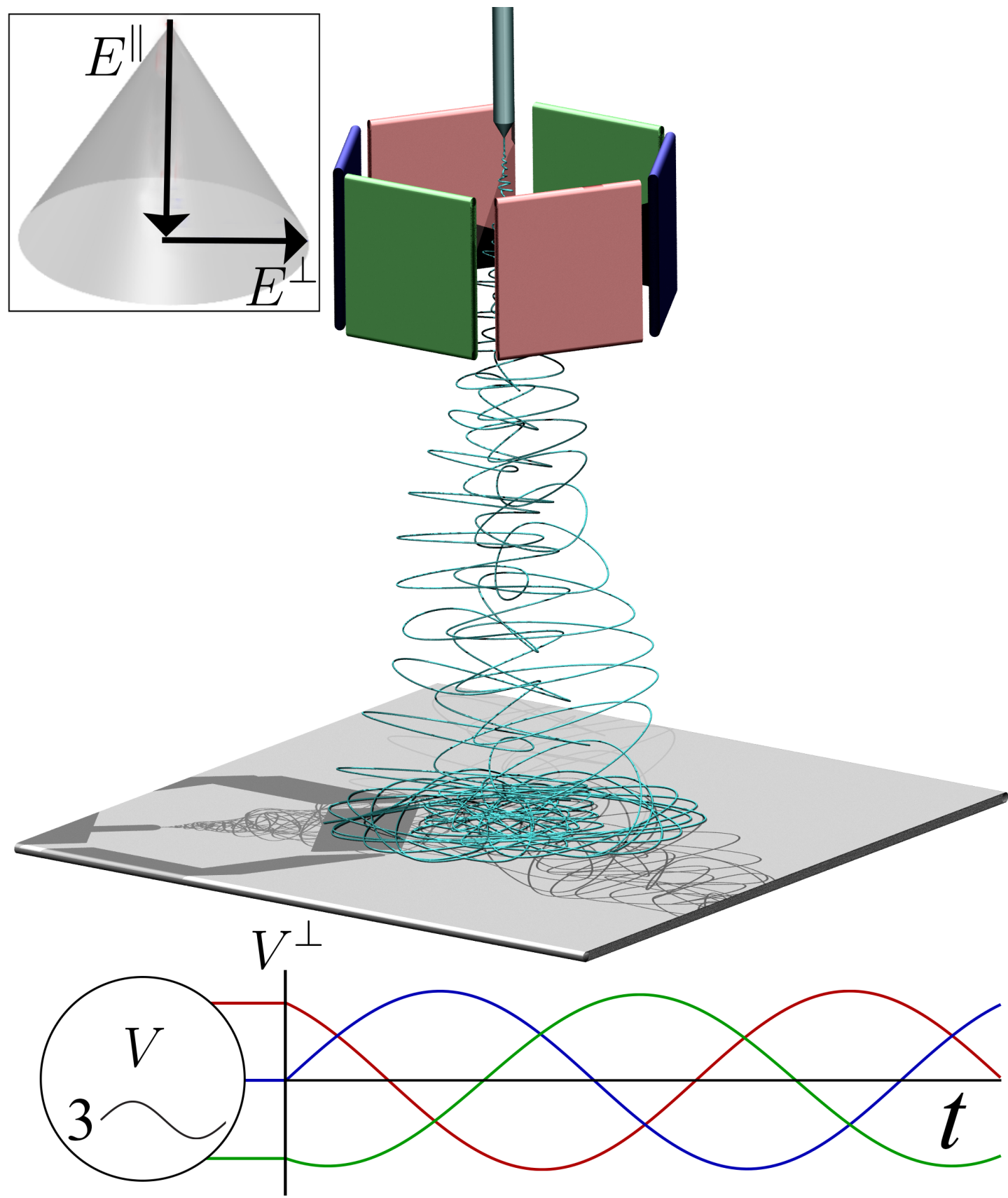

Figure 1. Sketch representation of the electrospinning process in presence of an orthogonal rotating electric field (OREF). The jet, ejected from a nozzle, is stretched by an electric field $E_{\|}$parallel to the $x$ axis. The OREF $E_{\perp}$ can be generated by a series of capacitor plates hexagonally arranged, and connected to a three-phase power source. The electrical power source is graphically represented in the bottom part of the figure. Here, the three phase voltage show colors corresponding to the color of the connected capacitor plate. 
Table I. Parameters values in the simulations.

\begin{tabular}{lll}
\hline \hline simulated time & 0.1 & $\mathrm{~s}$ \\
discretization length step $l_{\text {step }}$ & 0.02 & $\mathrm{~cm}$ \\
initial jet radius $R_{0}$ & $5 \cdot 10^{-3}$ & $\mathrm{~cm}$ \\
charge density & $4.4 \cdot 10^{4}$ & $g^{\frac{1}{2}} \mathrm{~cm}^{-\frac{3}{2}} \mathrm{~s}^{-1}$ \\
fluid viscosity $\mu$ & 20 & $\mathrm{~g} \mathrm{~cm}^{-1} \mathrm{~s}^{-1}$ \\
elastic modulus $G$ & $5 \cdot 10^{4}$ & $\mathrm{~g} \mathrm{~cm}^{-1} \mathrm{~s}^{-2}$ \\
collector distance $h$ & 16 & $\mathrm{~cm}$ \\
external electric potential $\left(h E^{\|}\right)$ & 30.021 & $\mathrm{~g}^{\frac{1}{2}} \mathrm{~cm}^{\frac{1}{2}} \mathrm{~s}^{-1}$ \\
surface tension $\alpha$ & 21.13 & $\mathrm{~g} \mathrm{~s}$ \\
bulk velocity $v_{s}$ & 0.28 & $\mathrm{~cm}^{-1}$ \\
perturbation frequency $\omega_{\text {pert }}$ & $10^{4}$ & $\mathrm{~s}^{-1}$ \\
perturbation amplitude $A_{\text {pert }}$ & $10^{-3}$ & $\mathrm{~cm}^{-3}$ \\
OREF modulus $A$ & {$[0.0,10.0]$} & $\mathrm{g}^{\frac{1}{2}} \mathrm{~cm}^{-\frac{1}{2}} \mathrm{~s}^{-1}$ \\
OREF frequency $\omega$ & {$[0.5,20.0]$} & $10^{4} \mathrm{~s}^{-1}$ \\
\hline \hline
\end{tabular}

\section{THE MODEL}

The jet emitted by the nozzle is modeled by a finite set of $n$ parcels at a distance $l_{i}$ (distance between the $i$ th and $(i+1)$ th parcel), connected via viscoelastic elements, similarly

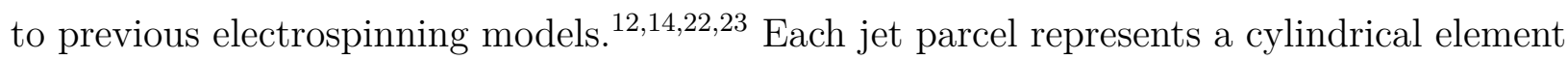
of jet volume $V_{i}=V_{0}$ and initial height $l_{i}=l_{\text {step }}$ (initial length step of jet discretization). As a consequence, the initial radius $R_{0}$ of the jet element is equal to $\sqrt{V_{0} / \pi l_{\text {step }}}$. From this representation, the following set of equations of motion (EOM) can be written for each parcel $i$ (hereafter, we shall consider the $x$-axis pointing from the nozzle to the collector): 


$$
\begin{aligned}
& \frac{d \vec{r}_{i}}{d t}=\vec{v}_{i} \\
& \frac{d \sigma_{i}}{d t}=\frac{G}{l_{i}} \frac{d l_{i}}{d t}-\frac{G}{\mu} \sigma_{i}, \\
& m_{i} \frac{d \vec{v}_{i}}{d t}=q_{i} \vec{E}+\sum_{j \neq i}\left(\frac{q_{i} q_{j}}{\left|\vec{r}_{j}-\vec{r}_{i}\right|^{2}} \vec{u}_{i, j}\right)-\pi R_{i}{ }^{2} \sigma_{i} \vec{t}_{i} \\
& +\pi R_{i+1}{ }^{2} \sigma_{i+1} \vec{t}_{i+1}+k_{i} \pi\left(\frac{R_{i}+R_{i+1}}{2}\right)^{2} \alpha \vec{c}_{i},
\end{aligned}
$$

In the above, the subscript $i$ stands for the $i$-th parcel, $\vec{r}_{i}$ is the position vector, $\vec{v}_{i}$ is the velocity vector, $G$ is the elastic modulus, $\mu$ is the viscosity of the fluid jet, $\sigma$ is the stress, $R$ is the cross sectional radius, $q$ is the charge, $\vec{E}$ is the electric field (Figure 1 ), $\vec{u}_{i, j}$ is the unit vector from parcel $i$ to parcel $j, \vec{t}_{i}$ is the unit vector pointing from parcel $i$ to $(i-1), k_{i}$ is the local curvature, $\alpha$ is the surface tension coefficient and $\vec{c}_{i}$ is the unit vector pointing from the parcel $i$ to the local centre of curvature. It is worth stressing that the filament radius $R_{i}$ at each $i$-th parcel location is equal to $\sqrt{V_{0} / \pi l_{i}}$, as a result of the volume conservation. Note that the constitutive Eq 2 models a Maxwell material with constant viscosity, in line with the approach of Refs ${ }^{1222}$.

The aforementioned system of EOM is numerically solved, starting each simulation with only two bodies: a parcel fixed at $x=0$, representing the spinneret nozzle, and a second parcel modeling the initial jet segment located at distance $l_{\text {step }}$ from the nozzle along the $x$ axis with a given initial velocity $v_{i}^{\circ}$ (defined below). Once the last parcel reaches a distance $2 l_{\text {step }}$ away from the nozzle, a new parcel (third body) is placed at a distance $l_{\text {step }}$, the length step used to discretize the jet as a sequence of parcels. Repeating this injection rule over time, we obtain a set of $n$ discrete jet elements.

The initial velocity is defined as $v_{i}^{\circ}=v_{s}+v_{d, i}$, where $v_{s}$ is a velocity term along the $x$ axis, modelling the bulk fluid velocity in the syringe needle, and $v_{d, i}$ denotes the dragging velocity

$$
v_{d, i}=\frac{v_{i-1}-v_{s}}{2} .
$$

The extra term $v_{d, i}$ accounts for the drag effect of the electrospun jet on the last inserted segment $(i-1)$. Note that the definition of $v_{d, i}$ was chosen in such a way as to keep the 
velocity strain unchanged before and after the parcel insertion.

Furthermore, we take into account a periodic nozzle perturbation with frequency $\omega_{\text {pert }}$ and amplitude $A_{\text {pert }}$, which models fast mechanical oscillations nearby the spinneret. This perturbation results in the emission of a conic helix jet. We are interested in adding an OREF to the above configuration, namely:

$$
\vec{E}=\vec{E}^{\|}+\vec{E}^{\perp}
$$

Hereafter, the main electric field and the OREF are denoted it by $\vec{E}^{\|}=\left(E_{x}, 0,0\right)$ and $\vec{E}^{\perp}=\left(0, E_{y}, E_{z}\right)$, respectively. In equations:

$$
\begin{aligned}
& E_{y}(A, \omega, t)=A \cos \omega t, \\
& E_{z}(A, \omega, t)=A \sin \omega t,
\end{aligned}
$$

where $A\left(\mathrm{~g}^{\frac{1}{2}} \mathrm{~cm}^{-\frac{1}{2}} \mathrm{~S}^{-1}\right)$ is the modulus, $\omega\left(s^{-1}\right)$ is the angular frequency. OREFs have already been treated in the literature, in the context of plasma confinement by means of a series of capacitor plates with alternating current around the apparatus. ${ }^{24}$ In JETSPIN, we modified the EOM according to equation (5)-(6).

With the exception of $A$ and $\omega$, in all simulations we use the numerical parameters proposed in Ref. 20 . These values were assessed by comparing with experimental data from an electrospinning process of polyvinylpyrrolidone (molecular weight $=1300 \mathrm{kDa}$, mixture of ethanol and water 17:3 v:v, with PVP concentration $2.5 \mathrm{wt} \%){ }^{[25}$ In particular, we consider the surface tension $\alpha=21.1 \mathrm{~g} \mathrm{~s}^{-2}$ from Ref. 26 , the elastic modulus $G=5 \cdot 10^{4} \mathrm{~g} \mathrm{~cm}^{-1} \mathrm{~s}^{-2}$ from Ref. .27 , and the shear viscosity $\mu=100 \mu_{0}$, where $\mu$ is taken equal to two orders of magnitude larger than the zero-shear viscosity $\mu_{0}=0.2 \mathrm{~g} \mathrm{~cm}^{-1} \mathrm{~s}^{-1}$, see Ref.26|28. This is because the strong longitudinal flows we are dealing with, can lead to an increase of the extensional viscosity, as observed in the literature.12129 Finally, $v_{s}$ is taken equal to 0.28 $\mathrm{cm} \mathrm{s}^{-1}$ which corresponds to a constant flow rate of $2 \mathrm{~mL} \mathrm{~h}^{-1}$ in a needle of radius $250 \mu \mathrm{m}$. The values of the simulation parameters are summarized in Table I

\section{RESULTS}

In the following, we present an exploratory study of the effects of the amplitude $A$ and frequency $\omega$ on the electro-spinning process. We wish to emphasize that this does not 
represent a fully-fledged analysis of the non-linear dynamical behaviour of this complex system, but rather a computational identification of the most interesting regions in the $A-\omega$ parameter space.

\section{A. Modulus and frequency of OREF}

We investigate the effect of $\vec{E}^{\perp}$ in equations (6), compared to the standard case $\vec{E}^{\|}$, and study the way that the jet morphology is deformed for different choices of the freeparameters. We take $A^{*}=\left|\vec{E}^{\|}\right| \simeq 1.8763 \mathrm{~g}^{\frac{1}{2}} \mathrm{~cm}^{-\frac{1}{2}} \mathrm{~S}^{-1}$ and $\omega^{*}=\omega_{\text {pert }}=10^{4} \mathrm{~s}^{-1}$ as a reference values, respectively for $A$ and $\omega$ in equations (6). A wide range of physically relevant values of these parameters is spanned by selecting $A \in[0,10]$ and $10^{-4} \omega \in[0.5,20]$ (see Ref. ${ }^{25}$ ). Such high values of the angular frequency $\omega$ mediate the OREF along the circumference orthogonal to $\vec{E}^{\|}$. Note that the dominant component of the force acting on each parcel is the one given by $\vec{E}^{\|}$, since the jet travels towards the collector without undergoing any breakup, such as that reported in literature.

The two components of the electric field are shown in the top left panel of Figure 1. where the red and blue arrows represent $\vec{E}^{\|}$and $\vec{E}^{\perp}$, respectively, while the total field $\vec{E}$ runs over the transparent grey conic surface. We run several simulations, each for a total time of $0.1 \mathrm{~s}$. After an initial drift $t_{\mathrm{drift}}=0.02 \mathrm{~s}$, the jet dynamics is assumed regular so that we can estimate mean values of suitable observables over their stationary statistical distributions. ${ }^{20}$ In particular, the mean value of the filament radius $R$ was estimated at the collector in different simulations with several pairs of $A$ and $\omega$ values. The surface of the mean radius $R$ was reconstructed as a function of $A$ and $\omega$ by linear interpolation, shown by contour lines in Figure 2. It is worth observing that for $A=0$ the mean cross section radius reduces to $R_{0}=4.05 \cdot 10^{-4} \mathrm{~cm}$, in agreement with the mean cross section values obtained for the standard case, $\vec{E}=\vec{E}^{\|}$. Further, as a representative value, we analyze the

case $A=5 \mathrm{~g}^{\frac{1}{2}} \mathrm{~cm}^{-\frac{1}{2}} \mathrm{~S}^{-1}$ in Figure 3, namely the red vertical line in Figure 2. Along such a line, we plot $R$ as a function of $\omega$, together with the corresponding confidence interval (namely the interquartile interval). In this figure, the thick black horizontal line indicates $R_{0}$ allowing a direct comparison between the cases with and without OREF.

From this plot, an oscillatory behavior of $R$ as a function of $\omega$ is clearly recognizable. Two representative points are singled out, namely the first minimum $m_{1}$, with $R\left(m_{1}\right)=$ 


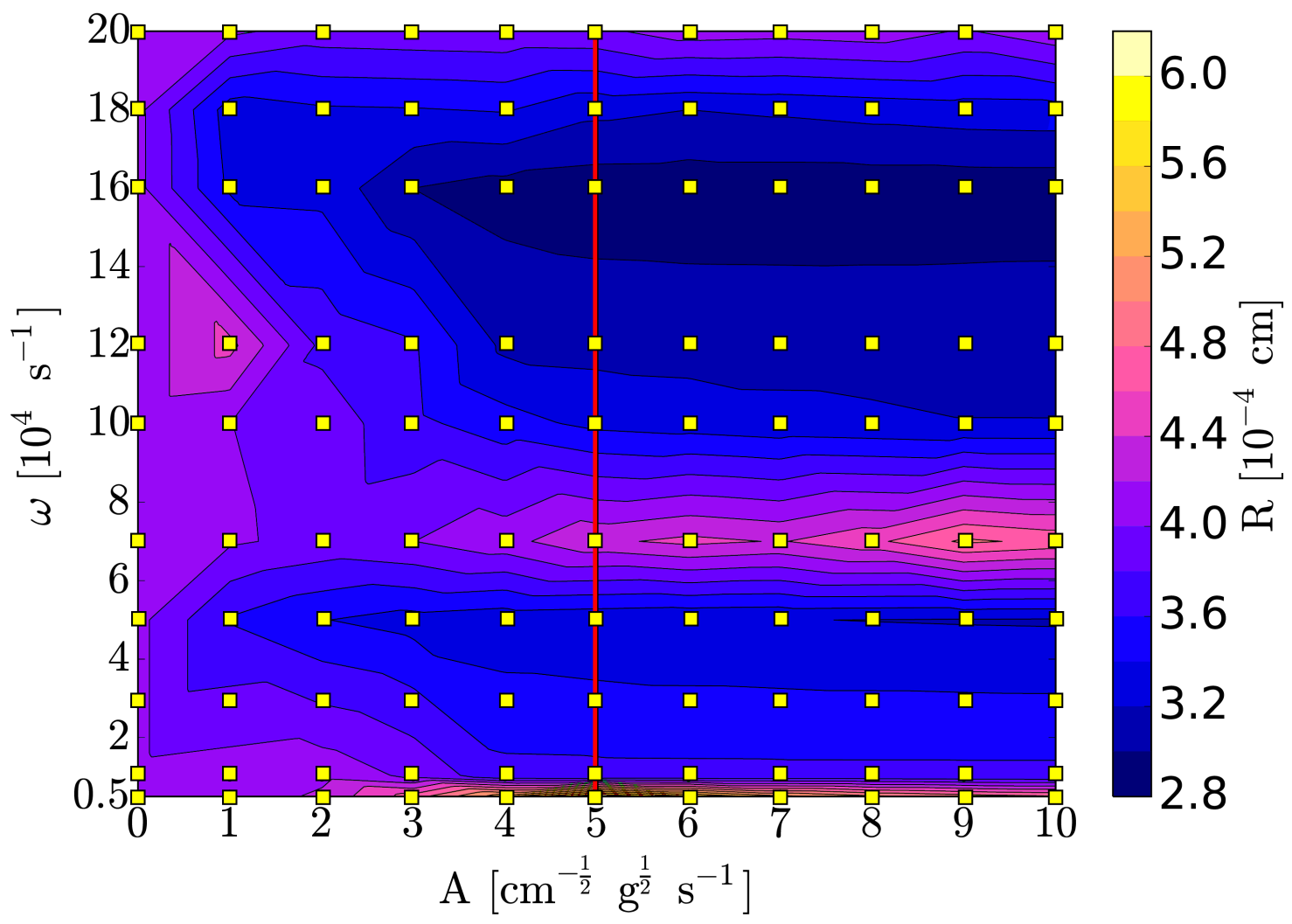

Figure 2. Contour lines showing the simulated mean jet radius $R$ at the collector as a function of $A \in[0,10]$ and $\omega \in[0.5,20]$ parameters. The $R(A, \omega)$ surface was assessed by a linear interpolation over a grid of pairs of $A$ and $\omega$, shown in figure as yellow square symbols.

$2.65 \cdot 10^{-4} \mathrm{~cm}$, corresponding to $\omega\left(m_{1}\right)=2.5 \times 10^{4} \mathrm{~s}^{-1}$ and the relative maximum $M_{1}$ with $R\left(M_{1}\right)=4.15 \cdot 10^{-4} \mathrm{~cm}$, with $\omega\left(M_{1}\right)=6 \times 10^{4} \mathrm{~s}^{-1}$. The corresponding jet-paths are reported in the sub-panels: one is the view from the side and the other is the view from the collector, looking up to the nozzle. It is worth noting that, although the helix associated with $\omega\left(M_{1}\right)$ is wider than the one for $\omega\left(m_{1}\right)$, the latter is more entangled, meaning that the jet undergoes longer-lived instabilities, resulting in a smaller value of $R$. 3D representations of the two highlighted trajectories are displayed in Figure 4, with color convention stated in the caption of Figure 3. A reduction of the cross-section by about $34 \%$ with respect to the case without OREF (namely $A=0$ in Figure 2 for which $R_{0}$ is reported) is observed. It is worth observing that such a reduction is obtained without altering the rheological properties of the jet by, say, changing the polymer concentration. This is comparable with similar results obtained by blowing-assisted electrospinning, where a gas stream, provided by suitable distributors 
around the nozzle, is employed as additional stretching force ${ }^{30 \mid} 32$ The latter technique was recently extended by Sinha-Ray et $A l \cdot 33$ to include supersonic blowing gaseous stream in electrospinning producing ultra-thin nanofibers.

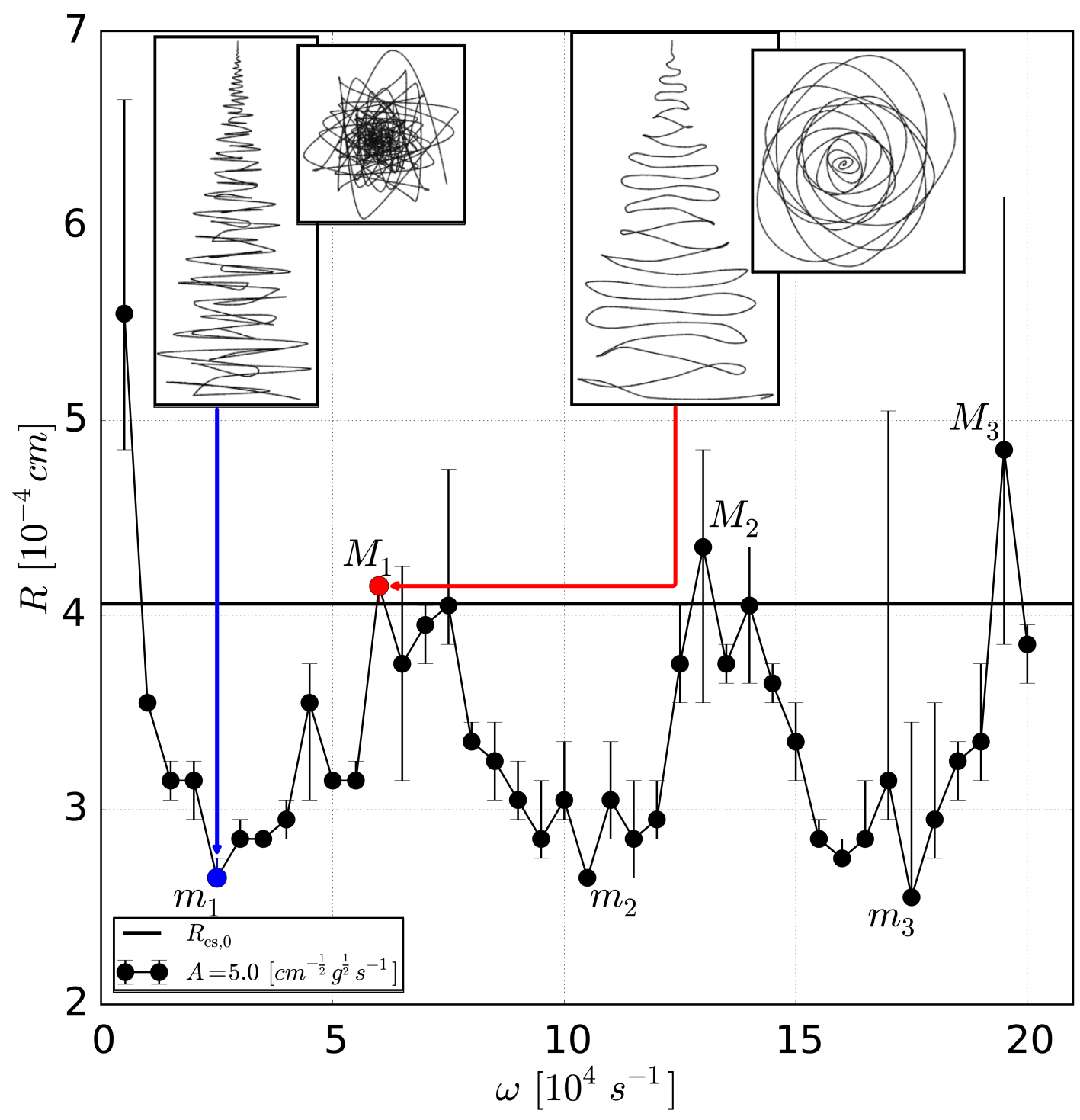

Figure 3. Mean of cross section radius $R$ at the collector for $A=5$ at several values of $\omega$, plotted with their respective interquartile as confidence intervals. The thick solid black horizontal line represents $R_{0}$, i.e. the mean value of $R$ in a standard electrospinning process (without OREF). The first minimum and maximum of $R$ are highlighted in red, with the insets showing the trajectories from the lateral and bottom planes.

Even though the functional relation $R=R(\omega)$ is the result of a highly complex structural dynamics, the oscillatory structure of such relation is relatively regular, and suggestive of a 


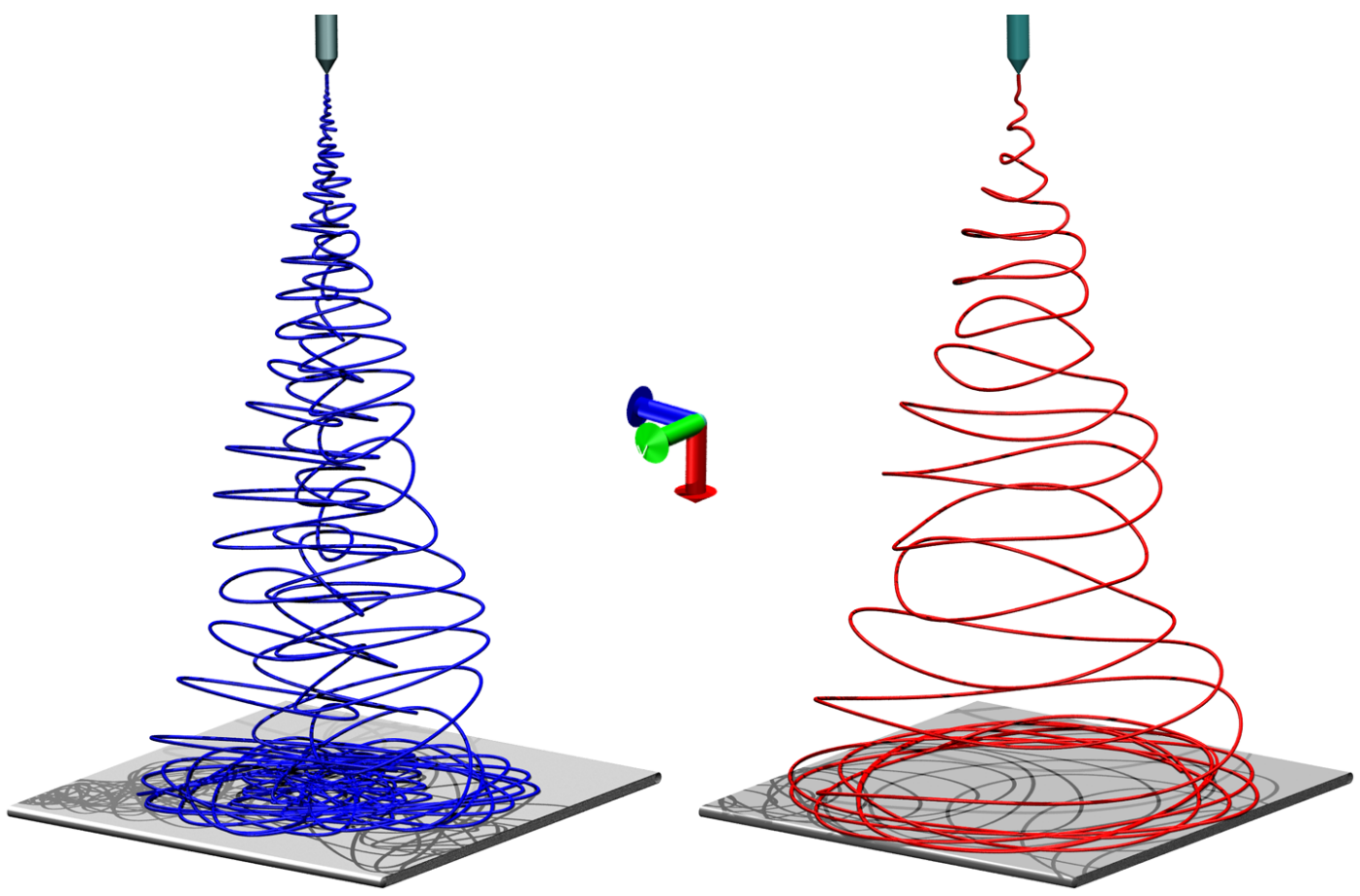

Figure 4. Jet trajectories with $A=5, \omega\left(m_{1}\right)=2.5 \cdot 10^{4} \mathrm{~s}^{-} 1$ (left blue curve) and $\omega\left(M_{1}\right)=6 \cdot 10^{4} \mathrm{~s}^{-} 1$ (right red curve). Axis convention, shown in the center, is as follows: red for $x$ axis, green for $y$ axis and blue for $z$ axis. While the red curve is wider than the blue one, the latter appears more twisted, thus producing a thinner $R$ (cfr with fig. 2 and 3 ).

sort of resonant mechanism underlying the OREF setup, which is likely to depend on the frequency $\omega_{\text {noz }}$ of the mechanical oscillation of the nozzle. Here, we have kept $\omega_{\text {noz }}$ at a fixed value, because it is practically easier to change the OREF frequency than modifying the inherent mechanical oscillations of the nozzle.

Nonetheless, we repeat the simulations corresponding to the two points $m_{1}$ and $M_{1}$ in Figure 3, with a nozzle perturbation $\omega_{n o z}=2.5 \cdot 10^{4} s^{-1}$. In both cases, we observe a change in the mean cross section radius $\langle R>$ between 5 and $10 \%$, compared to the previous values, confirming that both frequencies $\omega$ and $\omega_{\text {noz }}$ contribute to the oscillatory jet dynamics. At the moment, we have no clear theoretical explanation for such oscillatory behavior, which depends on the overall non-linear dynamics of the jet. However, we can define suitable observables which help elucidating the effects of the OREF on the jet morphology (see next Subsection). 


\section{B. Statistical analysis and overlap of trajectories}

Here, we provide both statistical and time-dependent analysis performed over the trajectories related to the most relevant $\omega$ values identified in the previous Subsection. Each jet trajectory is the result of a complex dynamics, which presents an initial drift in the time lapse where the filament has not yet reached the collector. After such time lapse, the trajectory regularizes and consequently it becomes possible to analyze the statistical distribution of the cross section radius.

The top part of Figure 5 shows normalized histograms for the distribution of cross section radius at the collector for three different frequencies, $\omega=[2.5,5.5,6] \cdot 10^{4} \mathrm{~s}^{-1}$, all with the same amplitude $A=5 \mathrm{~g}^{\frac{1}{2}} \mathrm{~cm}^{-\frac{1}{2}} \mathrm{~S}^{-1}$.

It is apparent that the frequency distribution is skewed and strongly non Gaussian, which is relatively unsurprising due to the highly non-linear nature of the process. Owing to this non-Gaussianity, this observable is best described via its median and confidence interval (given by the first and third quartiles), as reported in Figure 3. In order to clarify the trend of the mean radius reported in Figure 5 we define the jet length as:

$$
\Lambda(t)=\sum_{i=1}^{n-1}\left|\overrightarrow{\mathbf{r}}_{i+1}-\overrightarrow{\mathbf{r}}_{i}\right|,
$$

which measures the total length of the jet, from the collector up to the nozzle. Comparing the two trends of $R$ and $\Lambda$ in Figure 5, it is apparent that the two are anti-correlated, namely the smallest $\langle R\rangle$ corresponds to the longest $\langle\Lambda\rangle$ and vice versa. As a result, one of the main effects of OREF is to alter the jet path, which in turn modifies the jet stretch and the resulting cross sectional radius.

To gain further insights into the jet dynamics, it is also of interest to assess the "morphological distance" between two spirals corresponding to minimum and maximum fiber radii. Instead of a "smooth" Euclidean distance, we find it more informative to introduce an overlap distance between two trajectories $\alpha$ and $\beta$ by gaining insight from an order parameter usually exploited in the context of glassy materials (see Refs ${ }^{34}$ ), which is defined as follows

$$
Q_{\alpha \beta}(t, \varepsilon)=\int_{0}^{1} \Theta\left(\varepsilon-\left|\vec{r}_{\alpha}(t, \lambda)-\vec{r}_{\beta}(t, \lambda)\right|\right) d \lambda,
$$

In the above, $\lambda$ is the curvilinear coordinate, $\Theta$ the Heaviside step function, $\varepsilon(\mathrm{cm})$ a 

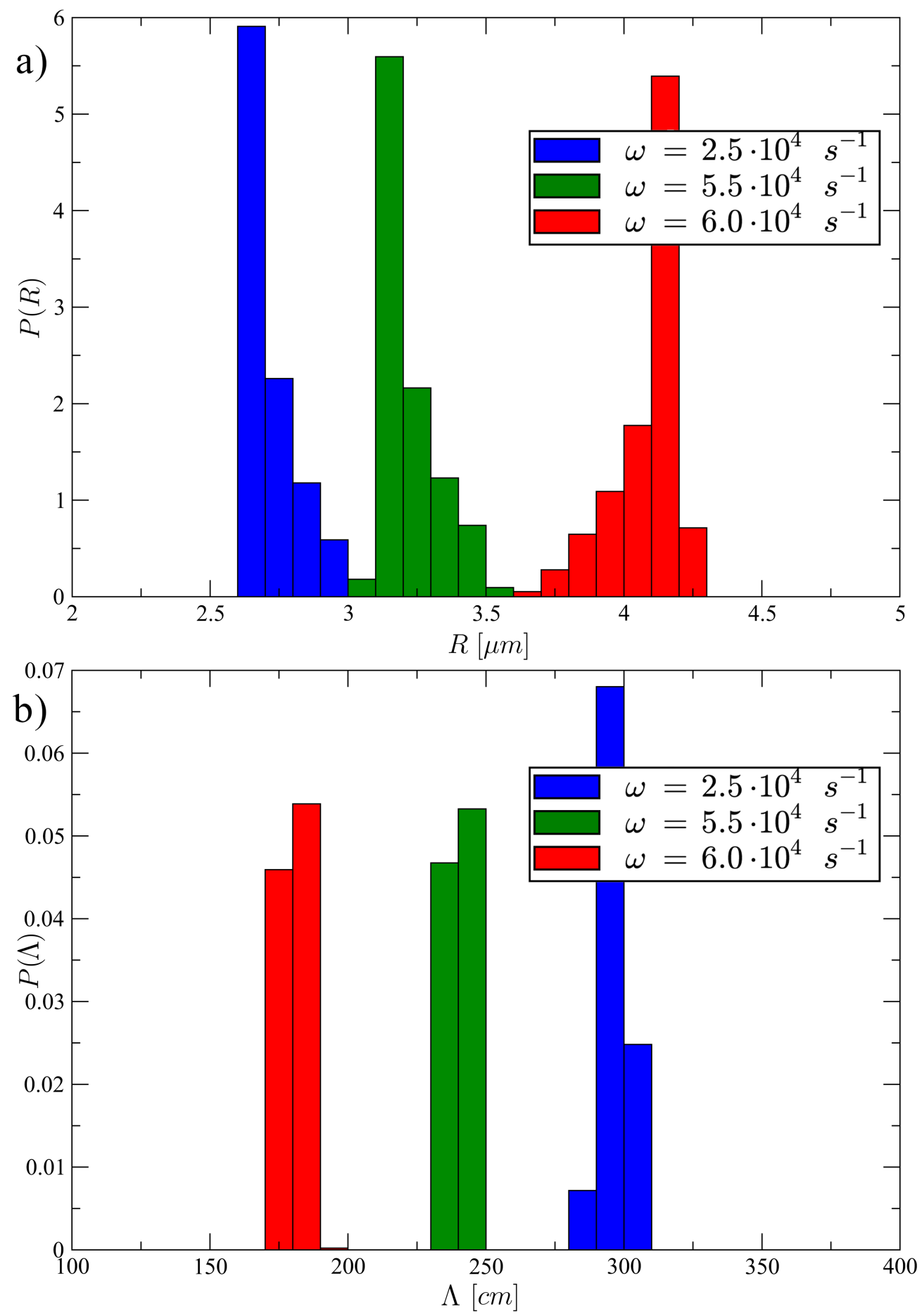

Figure 5. Top: the normalized histograms for distribution of cross section radius $R$ at the collector for $A=5$ and three values of $\omega$. Note that the distribution of the observable during the dynamics is strongly skewed and non-gaussian. Bottom: the corresponding normalized histograms for distribution of jet path length $\Lambda$, showing an opposite trend with respect to $<R>$. 
distance tolerance. The Heaviside step function acts like a switch, turning off whenever the distance of two homologue points with the same $\lambda$ at two different jets, goes above a given threshold $\varepsilon$. Therefore, $Q_{\alpha \beta}$ serves as a suitable indicator of the separation transition between two indistinguishable $(Q=1)$ and two fully separated $(Q=0)$ configurations, at the given scale $\varepsilon$. Note that by assuming the jet discretized as a set of $n$ parcels, each parcel can only contribute a factor $1 / n$ to the overlap parameter. This is in contrast with the Euclidean distance which may eventually be completely dominated by a single, localized, large deviation between the two jets. Being sensitive to the value of $\varepsilon, Q$ is a useful indicator of the dynamics of the separation process at different scales.

We evaluated the overlap distance for three values of the parameter $\varepsilon$ as a function of time $t$, and correlate this indicator with $R\left(M_{1}\right)$ and $R\left(m_{1}\right)$. As a reference value, we take $\varepsilon_{\text {ref }}=0.94 \mathrm{~cm}$, i.e. the pitch distance of the jet-path in the case $m_{1}$ (see sub-panel of Figure 3). Our results pertain to $\alpha \equiv m_{1}$ and $\beta \equiv M_{1}$, in Figure 6, where a separation transition between the two jets is apparent. By definition, for $\varepsilon=0$, the two jets are always separated, so that $Q_{m_{1} M_{1}}=0$ at all times.

On the other hand, at increasing $\varepsilon$, the two jets overlap only up to an initial time $t_{o v}$, while for $t>t_{o v} \sim 0.01 \mathrm{~s}$, a separation transition starts to take place, with the two jets getting more separated as $\varepsilon$ is made smaller. After $t=0.15 \mathrm{~s}$, the distance between the two configurations reaches its asymptotic value. On top of Figure 6, we report some snapshots of the two jets at a given time, to provide a visual counterpart of the corresponding values of $Q_{m_{1} M_{1}}(t, \varepsilon)$.

\section{Time series analysis of trajectories}

In order to gain further insight into the jet dynamics, we analyze the frequency spectra induced by OREF on the jet dynamics. In particular, the Fourier analysis exposes the frequencies of the swirling motion of the helix, which has a direct bearing on the jet path and the resulting fiber radius.

To this purpose, let us consider a plane perpendicular to the $x$ - axis placed at $x=8$ $\mathrm{cm}$, and denote it by $\vec{v}_{\perp}^{*}=v_{y}(\lambda \mid x=8 \mathrm{~cm}) \overrightarrow{u_{y}}+v_{z}(\lambda \mid x=8 \mathrm{~cm}) \overrightarrow{u_{z}}$ the projection on this plane of the velocity vector measured at the point $\lambda$ where the jet crosses such plane. 


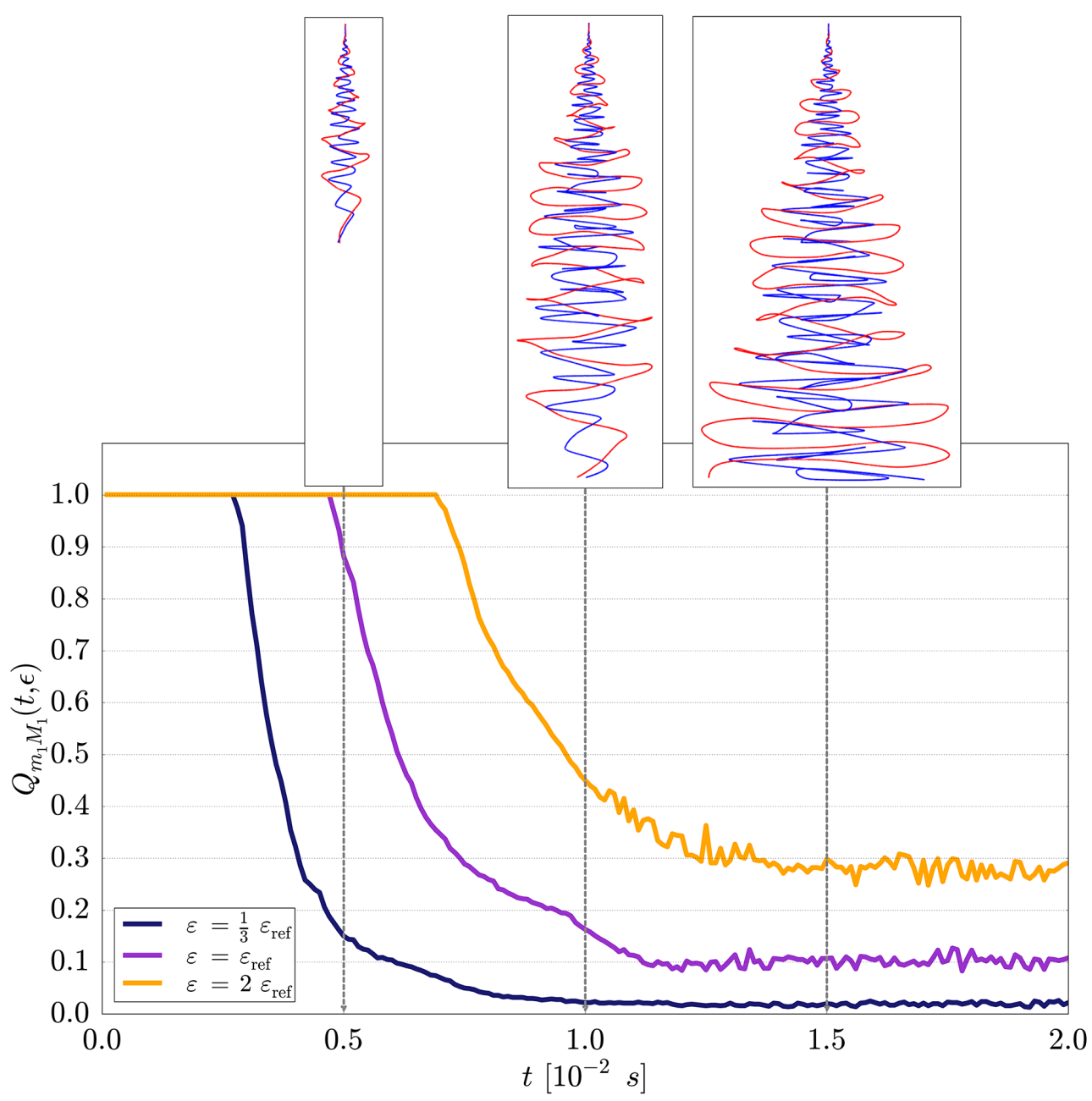

Figure 6. Overlapping function $Q_{m_{1} M_{1}}(t, \varepsilon)$ as a function of time, for three value of $\varepsilon$. For non-zero values of $\varepsilon$, an abrupt transition is evident, which stops after the drift time $t_{\text {drift }}$ has been reached. In particular, the smaller the value of $\varepsilon$, the smaller $Q(\varepsilon, t)$ is. Snapshots of the two jets analyzed at three times are reported at the insets over the plot, where color legend is the same as Fig. 4 We wish to remark that the trajectories should not self-intersect and they actually do not.

We inspect the normalized velocity autocorrelation function (VACF) of $\vec{v}_{\perp}^{*}$ defined as

$$
Z(\tau)=<\vec{v}_{\perp}^{*}(t) \cdot \vec{v}_{\perp}^{*}(t+\tau)>_{t} /<\vec{v}_{\perp}^{*}(t) \cdot \vec{v}_{\perp}^{*}(t)>_{t}
$$

where bracket denote time-averaging over the corresponding steady-state regime. The quantity $Z(\tau)$ measures the self-correlation of the swirling motion within the jet path. Then, cosine Fourier transformation (power spectra) of the VACF was computed for each OREF frequency, in order to expose the spectral densities of states. 37

We focus our attention on three main cases: first, the simulation setup without OREF 

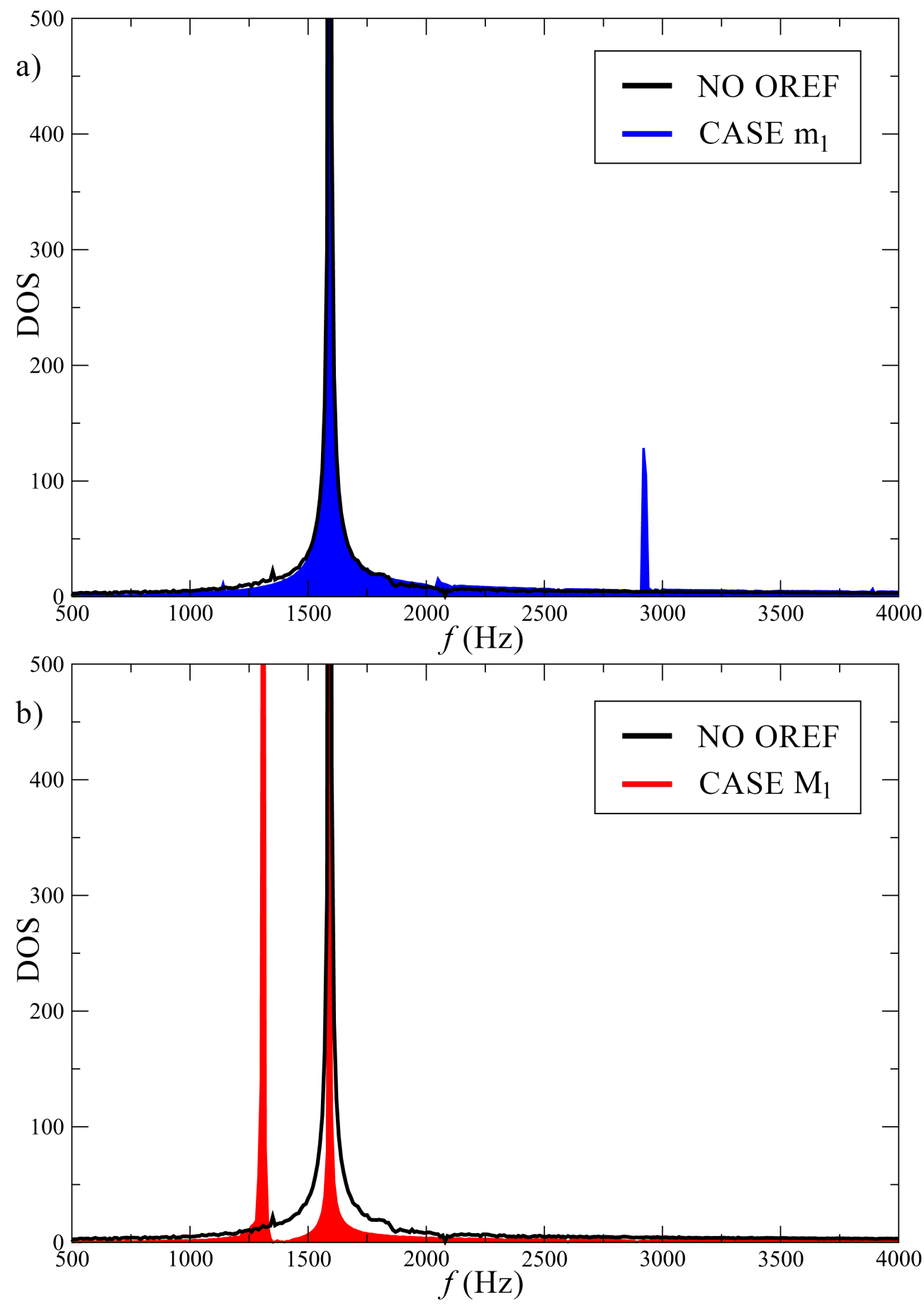

Figure 7. Cosine Fourier transformation (density of states) of the VACF computed for the three cases: without OREF as a reference case (drawn in black line), with OREF at $\omega\left(m_{1}\right)=2.5 \cdot 10^{4} \mathrm{~s}^{-1}$ (blue curve on top panel), and $\omega\left(M_{1}\right)=6 \cdot 10^{4} \mathrm{~s}^{-1}$ (red curve on bottom panel). We observe as the case $m_{1}$ corresponds to the presence of a second peak located at higher frequency, while the case $M_{1}$ is characterized by a second peak detected at lower frequency. 
as a reference case. The second and third correspond to the simulation setups denoted $m_{1}$ and $M_{1}$, respectively, in Figure 3. In Figure 7, we observe a central peak at $1600 \mathrm{~Hz}$ for all three cases under inspection, which is related to the main swirling motion resulting from the mechanical oscillation of the nozzle. Upon switching-on the OREF, satellite peaks are seen to appear, at higher(lower) frequencies for the case $m_{1}$ and $M_{1}$, respectively.

In order to elucidate the relation between the jet path $\langle\Lambda\rangle$ and the frequency of the secondary peak in the power spectra, we invoke simple arguments related to the helicoidal motion of jet.

For the sake of simplicity, we assume (neglecting other terms as Coulomb repulsive forces, etc.) that the centrifugal force of the $i-t h$ jet segment is approximately given by $F_{C, i} \propto q_{i} A$, where $A$ is the OREF amplitude, laid on the plane of rotation, and $q_{i}$ is the net charge of the jet segment. The corresponding curvature radius is $r_{c, i} \propto\left(q_{i} A\right) /\left(m_{i} \omega_{f}^{2}\right)$, where $m_{i}$ is the mass of the jet segment and $\omega_{f}$ is the angular swirling frequency reported in Figure 7 . Further, we can assess the pitch of the jet helix, defined as the height of a complete helix turn, $h_{i}=2 \pi v_{x, i} / \omega_{f}$, where $v_{x, i}$ is the velocity component of the $i$ - th jet segment along the $x$ axis.

A number of considerations on jet path and swirling frequency arise from the above relations. In particular, we note that the second peak in power spectra located at higher frequency provides a reduction of the helix pitch and an increase of the jet curvature, which is the reciprocal of the curvature radius. As a consequence, the number of helices drawn by the jet between the nozzle and the collector significantly increases, thereby providing a larger value of the jet path length $\Lambda$ observed in Figure 5. Furthermore, we observe that the OREF can also be used as a tool to control the jet curvature, and possibly drive the jet deposition on the collector, as detailed in the next Subsection.

\section{Statistical analysis of jet deposition at the collector}

The OREF significantly affects the spatial distribution of the jet, providing several patterns of the electrospun coatings deposited on the collector. In Figure 8, we report the normalized 2D maps, showing the probability of a jet parcel to hit the collector at the coordinates $y$ and $z$ (both perpendicular to $x$ by construction). Note that only the late dynamics describing the stationary regime was considered in order to compute the histograms. Here, 
we observe a clear modification of the pattern deposition as function of the applied OREF frequency $\omega$. In particular, by tuning the frequency, the deposition pattern is driven towards the inner region of the collector.

This is evident in top panel of Figure 8, where we report the normalized histogram for the case $m_{1}$, with the probability distribution spans over a precession motion. This is likely due to the combined effects of the two peaks in the frequency spectra (see Figure 7). Since the second peak is located at higher frequency, we observe a tighter deposition due to the larger jet curvature, depending on the frequency as shown in previous Subsection. On the other hand, if the second frequency lies below the one of the precession motion, the distribution stretches towards the external region of collector.

Therefore, the OREF frequency $\omega$ appears to offer new, possibly even time-dependent, strategies to achieve uniform distributions at the collector plate.

\section{SUMMARY AND OUTLOOK}

Summarizing, we have proposed the OREF mechanism and explored its effects on the electrospinning process, particularly on the radius of the electrospun fibers. Numerical simulations using the JETSPIN code show that such radius can be reduced up to about $30 \%$. Despite the inherent complexity of the underlying dynamics, the electrospinning response to OREF, $R=R(\omega)$ appears to organize into a rather regular oscillatory pattern, with periodic local minima and maxima of the finer radius as a function of the OREF frequency. The existence of such minima opens up the possibility of advancing electrospinning technologies and producing finer fibers with high repeatability. Further, the OREF can be used as a control mechanism to achieve uniform distributions of polymer filaments at the collector.

While a $30 \%$ reduction of the diameter of single nanofibers might not seem that dramatic, controlling the morphology of electrospun materials in such a finer way, might prove useful. For instance, the fraction of power of the fundamental mode of a cylindrical waveguide strongly (exponential-like) depends on the diameter of the guide, with a $30 \%$ variation of the diameter potentially leading to a significant (e.g., 20\%) change of coupled optical power. Also, fibers with such reduced size being most sensitive to their external environment, might lead to changes of their refractive index due to detected chemical or biological species, which can be probed by variations in the power transmitted in single nanofiber waveguides. Finally, 


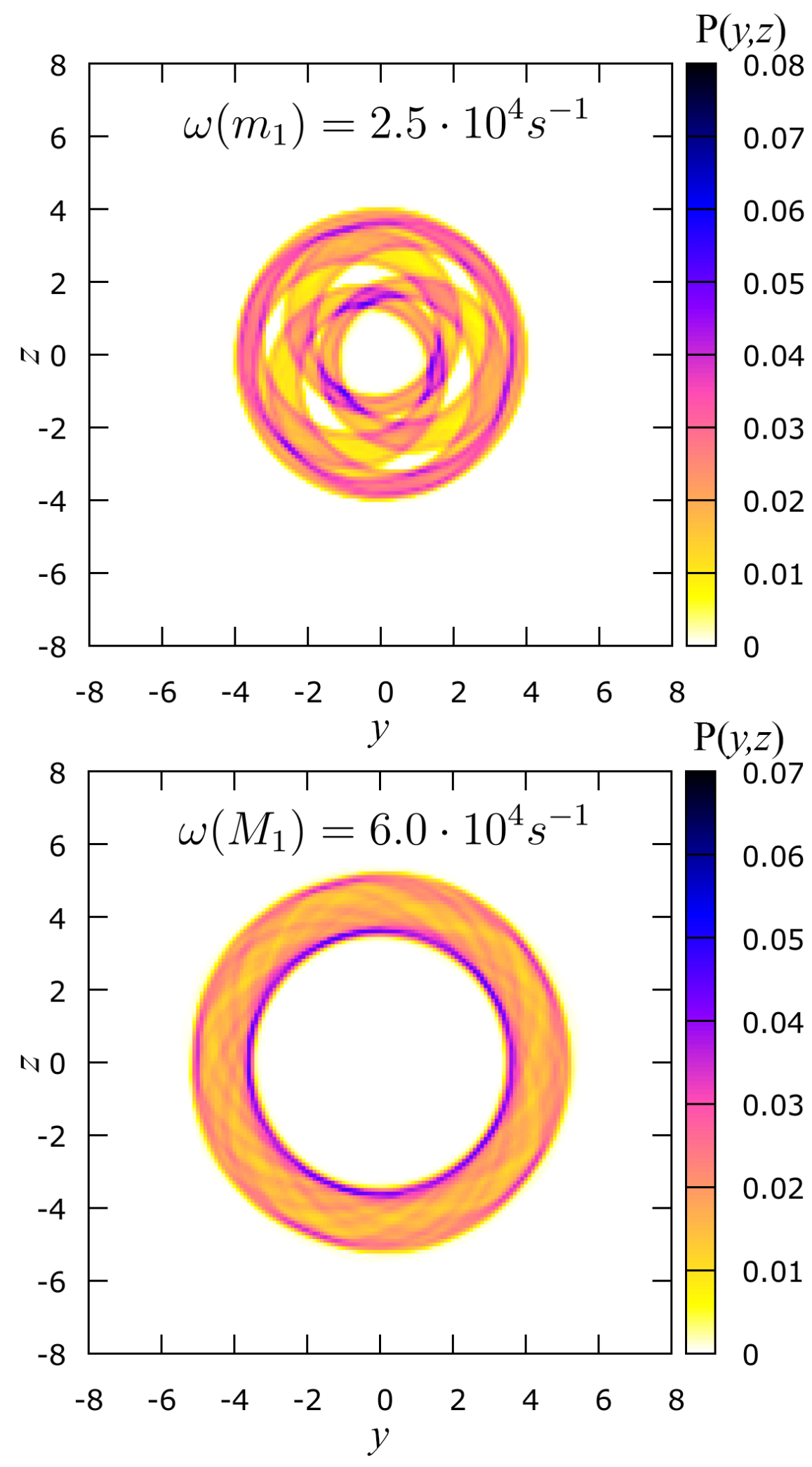

Figure 8. Normalized 2D maps computed over the coordinates $y$ and $z$ of the collector for the two cases with OREF at $\omega\left(m_{1}\right)$ (on top panel) and $\omega\left(M_{1}\right)$ (on bottom panel). The color palettes define the probability that a jet parcel hits the collector in coordinates $y$ and $z$. 
more uniform area coverage can lead to better coatings and improved filtrating materials. These perspectives are intriguing and worth experimental investigation.

The present simulations permit to highlight the salient morpho-dynamical features associated with the application of the OREF, as well as to probe the electrospinning response in a range of applied frequency. Much remains to be done for the future; particularly, the study of the spatial dependence of the self-consistent electrostatic field induced by charge deposition at the collector, and its effects on the overall jet dynamics and associated deposition patterns. Moreover, a fully-fledged analysis of the non-linear dynamical behavior of the OREF setup would be highly desirable. Studies along these lines are currently under way.

\section{ACKNOWLEDGMENTS}

The research leading to these results has received funding from the European Research Council under the European Union's Seventh Framework Programme (FP/2007-2013)/ERC Grant Agreement n. 306357 (NANO-JETS).

\section{REFERENCES}

${ }^{1}$ D. Li, Y. Wang, and Y. Xia, "Electrospinning nanofibers as uniaxially aligned arrays and layer-by-layer stacked films," Advanced Materials 16, 361-366 (2004).

${ }^{2}$ A. Greiner and J. H. Wendorff, "Electrospinning: a fascinating method for the preparation of ultrathin fibers," Angewandte Chemie International Edition 46, 5670-5703 (2007).

${ }^{3}$ C. P. Carroll and Y. L. Joo, "Axisymmetric instabilities of electrically driven viscoelastic jets," Journal of non-Newtonian fluid mechanics 153, 130-148 (2008).

4Z.-M. Huang, Y. Z. Zhang, M. Kotaki, and S. Ramakrishna, "A review on polymer nanofibers by electrospinning and their applications in nanocomposites," Composites science and technology 63, 2223-2253 (2003).

${ }^{5}$ A. L. Yarin, B. Pourdeyhimi, and S. Ramakrishna, Fundamentals and Applications of Micro and Nanofibers (Cambridge University Press, 2014).

${ }^{6}$ J. H. Wendorff, S. Agarwal, and A. Greiner, Electrospinning: materials, processing, and applications (John Wiley \& Sons, 2012). 
${ }^{7}$ D. Pisignano, Polymer Nanofibers: Building Blocks for Nanotechnology, 29 (Royal Society of Chemistry, 2013).

${ }^{8}$ A. L. Yarin, S. Koombhongse, and D. H. Reneker, "Bending instability in electrospinning of nanofibers," Journal of Applied Physics 89, 3018-3026 (2001).

${ }^{9}$ M. M. Hohman, M. Shin, G. Rutledge, and M. P. Brenner, "Electrospinning and electrically forced jets. i. stability theory," Physics of Fluids 13, 2201-2220 (2001).

${ }^{10}$ A. M. Ganan-Calvo, "On the theory of electrohydrodynamically driven capillary jets," Journal of Fluid Mechanics 335, 165-188 (1997).

${ }^{11}$ A. Huebner and H. Chu, "Instability and breakup of charged liquid jets," Journal of Fluid Mechanics 49, 361-372 (1971).

${ }^{12}$ D. H. Reneker, A. L. Yarin, H. Fong, and S. Koombhongse, "Bending instability of electrically charged liquid jets of polymer solutions in electrospinning," Journal of Applied physics 87, 4531-4547 (2000).

${ }^{13}$ M. Lauricella, D. Pisignano, and S. Succi, "Three-dimensional model for electrospinning processes in controlled gas counterflow," The Journal of Physical Chemistry A 120, 48844892 (2016).

${ }^{14}$ C. P. Carroll and Y. L. Joo, "Discretized modeling of electrically driven viscoelastic jets in the initial stage of electrospinning," Journal of Applied Physics 109, 094315 (2011).

${ }^{15}$ A. M. Ganan-Calvo, J. C. Lasheras, J. Dávila, and A. Barrero, "The electrostatic spray emitted from an electrified conical meniscus," Journal of Aerosol Science 25, 1121-1142 (1994).

${ }^{16}$ W. Yang, H. Duan, C. Li, and W. Deng, "Crossover of varicose and whipping instabilities in electrified microjets," Physical review letters 112, 054501 (2014).

${ }^{17}$ S. N. Reznik and E. Zussman, "Capillary-dominated electrified jets of a viscous leaky dielectric liquid," Physical Review E 81, 026313 (2010).

${ }^{18}$ D. H. Reneker and A. L. Yarin, "Electrospinning jets and polymer nanofibers," Polymer 49, 2387-2425 (2008).

${ }^{19}$ S. V. Fridrikh, J. H. Yu, M. P. Brenner, and G. C. Rutledge, "Controlling the fiber diameter during electrospinning," Physical review letters 90, 144502 (2003).

${ }^{20}$ M. Lauricella, G. Pontrelli, I. Coluzza, D. Pisignano, and S. Succi, "Jetspin: A specificpurpose open-source software for simulations of nanofiber electrospinning," Computer Physics Communications 197, 227-238 (2015). 
${ }^{21}$ M. Lauricella, G. Pontrelli, D. Pisignano, and S. Succi, "Dynamic mesh refinement for discrete models of jet electro-hydrodynamics," Journal of Computational Science 17, 325333 (2016).

${ }^{22}$ T. A. Kowalewski, S. Sbłonski, and S. Barral, "Experiments and modelling of electrospinning process," Technical Sciences 53 (2005).

${ }^{23}$ Y. Sun, Y. Zeng, and X. Wang, "Three-dimensional model of whipping motion in the processing of microfibers," Industrial \& Engineering Chemistry Research 50, 1099-1109 (2010).

${ }^{24}$ X. P. Huang, F. Anderegg, E. M. Hollmann, C. F. Driscoll, and T. M. O'neil, "Steady-state confinement of non-neutral plasmas by rotating electric fields," Physical Review Letters 78, 875 (1997).

${ }^{25}$ M. Montinaro, V. Fasano, M. Moffa, A. Camposeo, L. Persano, M. Lauricella, S. Succi, and D. Pisignano, "Sub-ms dynamics of the instability onset of electrospinning," Soft matter 11, 3424-3431 (2015).

${ }^{26}$ N. Yuya, W. Kai, B. S. Kim, and I. S. Kim, "Morphology controlled electrospun poly(vinyl pyrrolidone) fibers: effects of organic solvent and relative humidity," Journal of Materials Science and Engineering with Advanced Technology.

${ }^{27}$ V. N. Morozov and A. Y. Mikheev, "Water-soluble polyvinylpyrrolidone nanofilters manufactured by electrospray-neutralization technique," Journal of Membrane Science 403, 110-120 (2012).

${ }^{28} \mathrm{~V}$. Bühler, Polyvinylpyrrolidone excipients for pharmaceuticals: povidone, crospovidone and copovidone (Springer Science \& Business Media, 2005).

${ }^{29}$ A. L. Yarin, Free liquid jets and films: hydrodynamics and rheology (Longman Scientific \& Technical Harlow, 1993).

${ }^{30}$ X. Wang, I. C. Um, D. Fang, A. Okamoto, B. S. Hsiao, and B. Chu, "Formation of water-resistant hyaluronic acid nanofibers by blowing-assisted electro-spinning and nontoxic post treatments," Polymer 46, 4853-4867 (2005).

${ }^{31}$ Y. Lin, Y. Yao, X. Yang, N. Wei, X. Li, P. Gong, R. Li, and D. Wu, "Preparation of poly (ether sulfone) nanofibers by gas-jet/electrospinning," Journal of Applied Polymer Science 107, 909-917 (2008).

${ }^{32}$ H. Y. Hsiao, C. M. Huang, Y. Y. Liu, Y. C. Kuo, and H. Chen, "Effect of air blowing on the morphology and nanofiber properties of blowing-assisted electrospun polycarbonates," 
Journal of Applied Polymer Science 124, 4904-4914 (2012).

${ }^{33}$ S. Sinha-Ray, M. W. Lee, S. Sinha-Ray, S. An, B. Pourdeyhimi, S. S. Yoon, and A. L. Yarin, "Supersonic nanoblowing: a new ultra-stiff phase of nylon 6 in 20-50 nm confinement," Journal of Materials Chemistry C 1, 3491-3498 (2013).

${ }^{34}$ M. Ozawa, W. Kob, A. Ikeda, and K. Miyazaki, "Equilibrium phase diagram of a randomly pinned glass-former," Proceedings of the National Academy of Sciences 112, 6914-6919 (2015).

${ }^{35}$ W. Kob and L. Berthier, "Probing a liquid to glass transition in equilibrium," Physical review letters 110, 245702 (2013).

${ }^{36}$ T. R. Kirkpatrick, D. Thirumalai, and P. G. Wolynes, "Scaling concepts for the dynamics of viscous liquids near an ideal glassy state," Physical Review A 40, 1045 (1989).

${ }^{37}$ M. P. Allen and D. J. Tildesley, Computer simulation of liquids (Oxford university press, 1989).

${ }^{38}$ V. Fasano, A. Polini, G. Morello, M. Moffa, A. Camposeo, and D. Pisignano, "Bright light emission and waveguiding in conjugated polymer nanofibers electrospun from organic salt added solutions," Macromolecules 46, 5935-5942 (2013). 